\title{
Effects of a Glutamine Supplement on the Skeletal Muscle Contractile Force of Mice
}

\author{
Derek Waddell and Kim Fredricks \\ Department of Biology \\ Viterbo University \\ 921 S. $7^{\text {th }}$ Street \\ La Crosse, Wisconsin 54601 USA
}

Received: June 6, 2005

Accepted: August 10, 2005

\begin{abstract}
L-glutamine is the most abundant amino acid found in human muscle and plays an important role in protein synthesis. It is, therefore, believed that by increasing L-glutamine levels, protein metabolism can be increased, resulting in skeletal muscle growth. The goal of this research was to determine if increased L-glutamine levels enhanced muscle growth in mice. Comparisons between the contractile force of the gastrocnemis muscles of a control group and a group that received the supplement through their water supply were intended to determine if muscle growth occurred. T-tests were used to compare the contractile forces of the experimental and control mice. There was a significant difference between the contractile forces of the experimental and control groups. This suggests that L-glutamine supplementation increased the muscle growth of the experimental mice.
\end{abstract}

\section{INTRODUCTION}

Athletes are continually searching for ways to improve their performance to provide them with an advantage over their opponent. Increasing one's speed or strength is the most common way athletes are able to give themselves an advantage over other competing athletes. Weightlifting is one way of improving an individual's physical fitness, but many athletes are not satisfied with the results from weightlifting alone and choose to use growth supplements to further increase their muscle development. Because steroids are illegal for athletes to use in most sports, alternative supplements, such as Nitrix, NOX2, and Primobolan have bombarded the nutritional market. Each of these new supplements is designed to increase muscle growth and development.
One current widely used supplement is L-glutamine, which increases protein synthesis within skeletal muscle leading to enhanced muscle growth. By increasing muscle mass, the contractile force of a muscle can be increased $[1,2]$. The mechanism used to describe the contraction of skeletal muscle is called the sliding filament mechanism. A muscle contracts because myofibrils within the muscle fibers slide in the opposite directions of one another [2]. An increase in muscle growth allows more myofibrils to be activated, which increases force of contraction [2].

Glutamine is a soluble white crystalline amino acid that is found in plant and animal tissues [3,4]. Glutamine is also the most abundant amino acid within the human body, and about $90 \%$ of the body's glutamine is within skeletal muscle $[5,6]$. It 


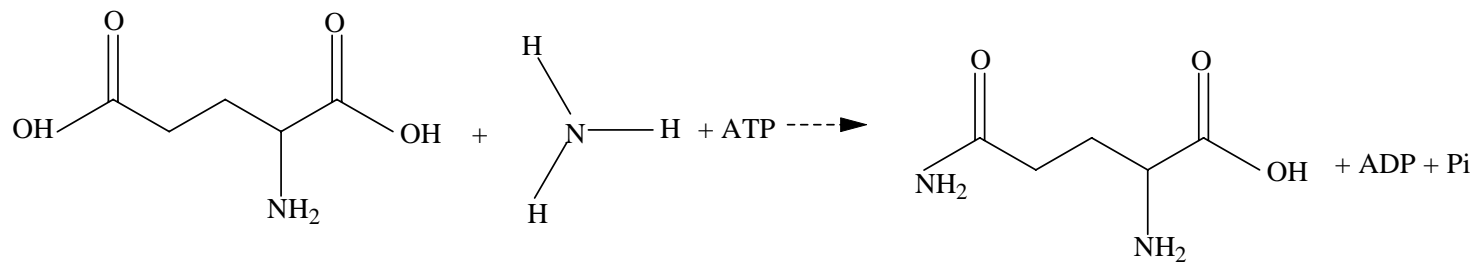

\section{Glutamate $+\mathbf{N H}_{3}+\mathrm{ATP} \longrightarrow$ Glutamine + ADP $+\mathbf{P}_{\mathbf{i}}$}

Figure 1. Synthesis of glutamine in the human body [6].

was first isolated in beet juice and wheat in 1883 , and it was believed by many that glutamine was a non-essential amino acid due to the abundance produced by the human body $[5,7]$. Throughout the evolution of the human body, glutamine production has persisted indicating that this supposed non-essential amino acid is required for maintaining specific functions within the human body, specifically within skeletal muscle $[5,8]$. Skeletal muscle synthesizes glutamine by combining glutamate, ammonia, and ATP (Figure 1) $[6,9,10]$.

Glutamine is especially important to the human body during times of stress caused by surgery or illness. This type of improved protein synthesis during times of stress has been documented in abundance $[8,11,12]$. After surgery, for example, glutamine levels within skeletal muscles decrease $[4,13,14]$. This decrease is accompanied by a decrease in muscle protein synthesis $[13,15]$. By increasing the patient's glutamine intake after surgery, protein synthesis is increased, leading to a quicker recovery. In contrast, the effect of increasing glutamine intake on protein synthesis within a normal healthy individual, such as a physically fit athlete, has not been well documented [8]. This research is intended to determine if a glutamine supplement leads to muscle growth, specifically skeletal muscle growth.

Skeletal muscles are voluntary striated muscles attached to one or more bones [2]. This means conscious control is required for movement to occur in a skeletal muscle. Branched throughout each skeletal muscle fiber are motor neurons responsible for delivering the electrical signals from the brain to the muscle. The contraction of a skeletal muscle consists of four phases: excitation, excitation-contraction coupling, contraction, and relaxation. The first step is for the muscle to be stimulated by a nerve. Action potentials in nerve fibers result in an action potential in muscle fibers during the excitation phase. Action potentials are fluctuations of voltage along cell membranes, and these action potentials travel along nerve fibers delivering signals throughout the body. During the excitationcontraction coupling phase, the muscle is prepared for contraction with the delivery of the action potential to the muscle. The muscle fibers develop tension during the contraction phase. It is during this phase that the myofilaments slide past each other. Muscle fibers relax and return to resting length during the relaxation phase. This occurs because the nerve stops sending action potentials. This movement of the skeletal muscle produces a myogram when attached to some type of recording device, such as Power Lab by ADInstruments (Figure 2) [2]. In addition, force of contraction can be determined from the myogram.

The strength of a muscular contraction is many times affected by simply the size of the muscle $[1,2,16]$. By determining the contractile force of the gastrocnemis muscle of the experimental and control mice, stimulated with a given voltage, the ability of a glutamine supplement to enhance the skeletal muscle growth of an individual can be determined. Specifically, this study tested the contractile forces produced by the mice that have consumed the glutamine supplement and 


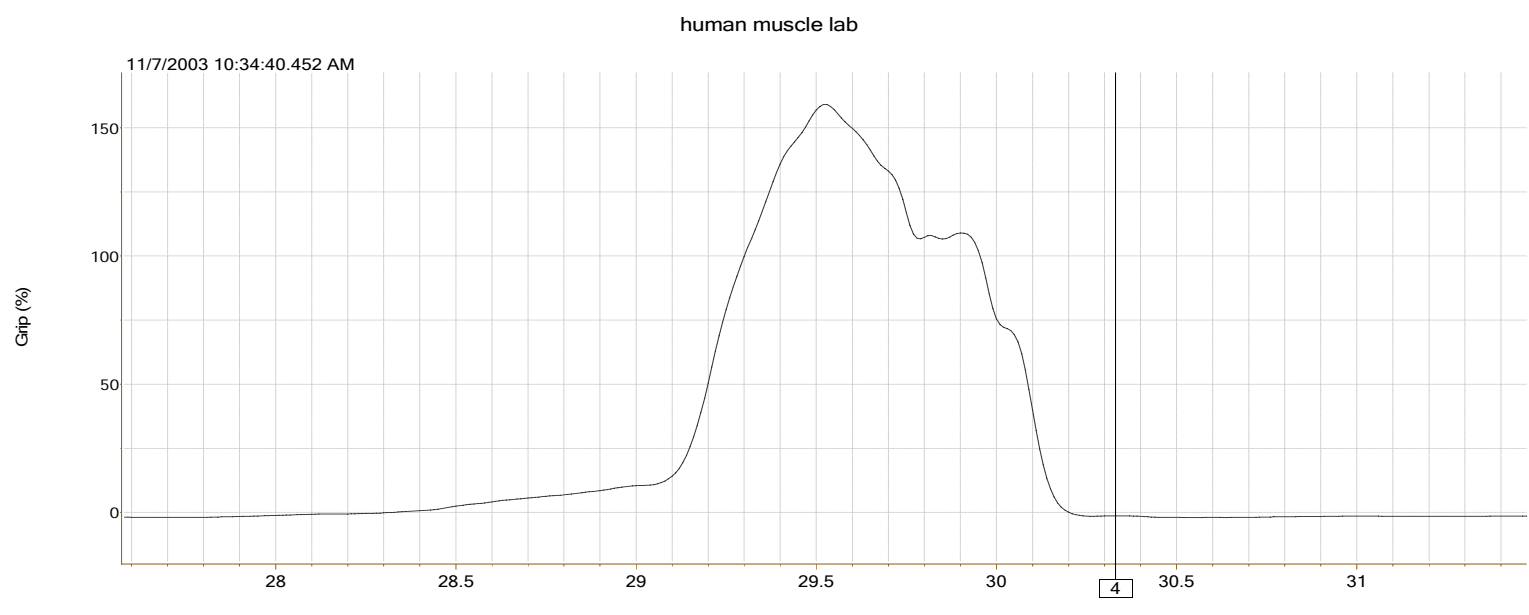

Figure 2. Myogram of a human muscle contraction produced by Power Lab software [2].

compared them to control mice. Our hypothesis was that the contractile forces exerted by the experimental mice would be greater than the control mice that did not consume any supplement.

\section{METHODS}

\section{a. Animal Care}

Twenty-two CF-1 male mice were obtained from Charles River Laboratories of Wilmington, Massachusetts. These mice were approximately eight weeks old upon arrival. The mice were housed in individual containers at room temperature $\left(26^{\circ} \mathrm{C} \pm 2\right.$ ${ }^{\circ} \mathrm{C}$ ) in the animal care room of the Reinhart Center at Viterbo University. Adequate amounts of food, Purina rodent chow, and water were supplied to the mice ad libitum. The bedding of the containers was changed weekly to ensure that the mice received proper levels of nutrition and sanitation. Each mouse was given fifteen pellets of food and $100 \mathrm{ml}$ of water every two days. The amount of food and water remaining were also measured every two days to determine the quantity of food and water each mouse consumed.

\section{b. Experimental Groups}

\section{Control Group}

The control group consisted of eleven male mice, which allowed sufficient amount of data to be generated for statistical power to be obtained. These mice received amounts of water and food that were equal to the amounts administered to the experimental group, except the water supply of the control mice consisted of only tap water. The weight of each control mouse was also recorded at the beginning of the experiment, and their final weights were recorded before their skeletal muscle contraction forces were measured.

\section{Experimental Group}

The experimental group again consisted of eleven male mice that were administered an L-glutamine supplement through their drinking water for four weeks. The L-glutamine (Sigma ${ }^{\circledR} \quad$ G-5763) was soluble in water allowing the L-glutamine to be effectively delivered in the $100 \mathrm{ml}$ water supply of each mouse. During the first week, the mice underwent a loading period in which the mice were given a stock fluid level of $11.2 \mathrm{mg} / \mathrm{ml}$ of supplement in their water supply [17]. After the loading period, the mice received a decreased dose of 5.6 $\mathrm{mg} / \mathrm{ml}$ of supplement for the next three weeks [17]. The amount of water consumed by each individual experimental mouse was again measured every two days to determine the amount of supplement consumed by each mouse. As with the control mice, the weight of each experimental mouse was also recorded at the beginning of the experiment, and their final weights were recorded before their skeletal muscle contraction forces were measured. 
a. Determination of Contractile force and Analyzing the Data

To determine the contractile force of the gastrocnemius muscles of the control and experimental mice, the Power Lab equipment and software produced by ADInstruments was used. First, the mice were euthanized with ether. This method of euthanization is the typical protocol that is followed to euthanize mice, and is considered a humane method of euthanization (Dr. K.T. Fredricks, pers. comm.). Once a mouse was euthanized, the skin of the right hind leg was removed exposing the gastrocnemius muscle. This muscle remained attached to the proximal end of the femur, but the calcaneal tendon was cut. The free end of the muscle was attached to the force transducer. Electrodes connected to the Power Lab set-up were then attached to the muscle to deliver a low level electrical shock that depolarized the muscle producing a contraction. These electrodes were placed approximately $1 \mathrm{~cm}$ apart depending on the length of the gastrocnemius muscle. A hook was attached to the Achilles tendon and to a force transducer by a short length tread. Slack was removed from the thread by adjusting the tension on the force transducer. Additionally the tread was kept perpendicular to the base of the force transducer. The force transducer measured the contractile force when an electrical stimulus was delivered to the muscle through the electrodes, and the muscle contracted. The Power Lab software recorded and graphed the muscle contractions in the form of a myogram. To determine the amount of voltage that was necessary to produce a maximum contraction of the gastrocnemius muscle of each mouse, a control mouse was used. The set up was as described above and stimulus intensity was slowly increased until a maximum contraction was reached. A voltage of $20 \mathrm{mV}$ was required for a maximum contraction to be obtained.

Once the contractile forces of both the control and experimental mice were recorded, one-tailed t-tests assuming unequal variances were used to determine if the contractile forces of the two treatments were significantly different. The t-tests were carried out at a 95\% confidence interval using Microsoft Excel software. A t-test value less than 0.05 proves a significant difference between the control and experimental groups existed.

III. RESULTS

Determining if L-glutamine supplementation produced a stronger contractile force in mice was accomplished by measuring the muscle contraction and weight gain of each control and experimental mouse examined. By dividing the contractile force of a mouse by its final weight the amount of force exerted can be measured in Newton's per gram. This calculation allows for differences in mouse size to be taken into consideration as a larger mouse could produce a larger force of contraction than a smaller mouse without the involvement of any supplementation. The average contractile force obtained from the eleven experimental mice, which were administered the L-glutamine supplement in their drinking water, was $0.0018 \mathrm{~N} / \mathrm{g}$ (Figure 3 ). The average contractile force obtained from the control mice, which consumed only tap water, was $0.0008 \mathrm{~N} / \mathrm{g}$ (Figure 3). The result of the t-test using these average contractile forces was a p-value of 0.0037 with significant results existing below a value of 0.05 .

The average weight gained by both the control and experimental mice was determined. The average amount of weight gained by the experimental mice was $2.4 \mathrm{~g}$ (Figure 4). The average amount of weight gained by the control mice was $1.66 \mathrm{~g}$ (Figure 4). The result from a one-tailed ttest assuming unequal variances using the average weight gain of the experimental and control groups was a p-value of 0.024 with significant results being less than a value of 0.05 .

The amounts of food and water consumed were also measured. One-tailed t-tests were used to determine if significant differences existed between the amounts consumed by the experimental and control mice weekly. There were no significant differences over the first two weeks of the experiment for food or water consumed. However, the experimental mice drank significantly more than the control mice during weeks three and four $(p=0.00008, p$ $=0.006$ ). 


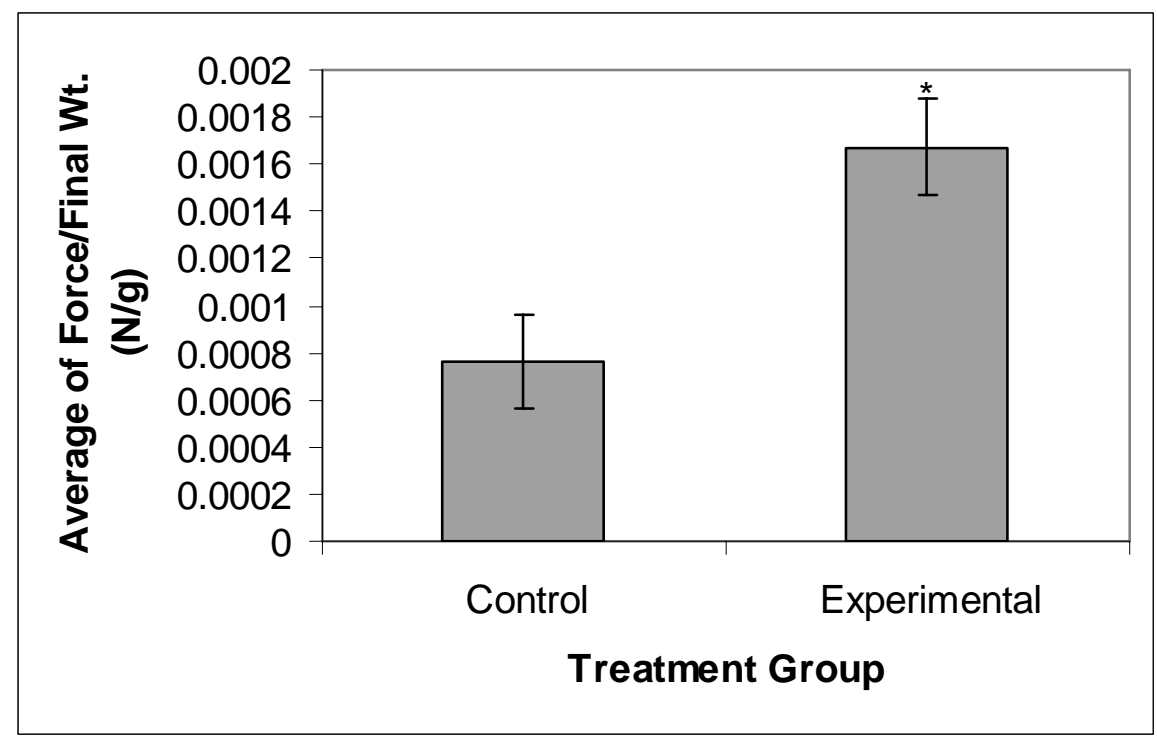

Figure 3. The average of the contraction force of the gastrocnemius muscle divided by the final weight of the mouse is depicted. Contraction forced was obtained by producing a maximum contraction with the application of electrical stimulus. *A significant difference existed between the control and experimental groups $(p<0.05)$.

\section{DISCUSSION}

The proposed hypothesis for this given research expected an increase in the skeletal muscle contractile force of experimental mice. From these results, we conclude that by administering an Lglutamine supplement to mice through their drinking water their skeletal muscle contractile forces were increased. With a greater contractile force, we could also conclude that the mice that received the Lglutamine experienced greater muscle growth. Large muscles produce greater forces of contraction due to the increased number of myofilaments available to create a muscle contraction [2]. The average contractile force normalized to body weight for the experimental mice was almost two times greater than the average contractile force of the control mice per grams of body weight (Figure 3). As the amount of muscle mass an individual possesses increases, the individual's weight also increases because of the additional mass. Both the control and experimental mice experienced weight gain during the four-week experiment due to normal development processes. The experimental mice, however, exhibited a significantly greater average weight gain than the control mice (Figure 4). This significant difference between the average weight gains of the experimental and control mice may have been due to an increase in muscle mass of the experimental mice. If the greater weight gain experienced by the experimental mice was caused by an increase in muscle mass, the supplementation of L-glutamine could have increased muscle development. Increased muscle development would, therefore, lead to a greater muscle contractile force in mice that consume an L-glutamine supplement than in mice that don't consume the supplement.

Because the mice did not consume significantly different amounts of food, the increase in weight can't be attributed to diet. The experimental mice did apparently consume more water, which could add weight. However, they could have brushed against the sipper tubes to cause excess water to leak out of the bottles. Differences in water consumption between the control and experimental groups could have also been attributed to the accumulation of bedding around the sipper tubes causing water to leak out of the bottles. We did find 


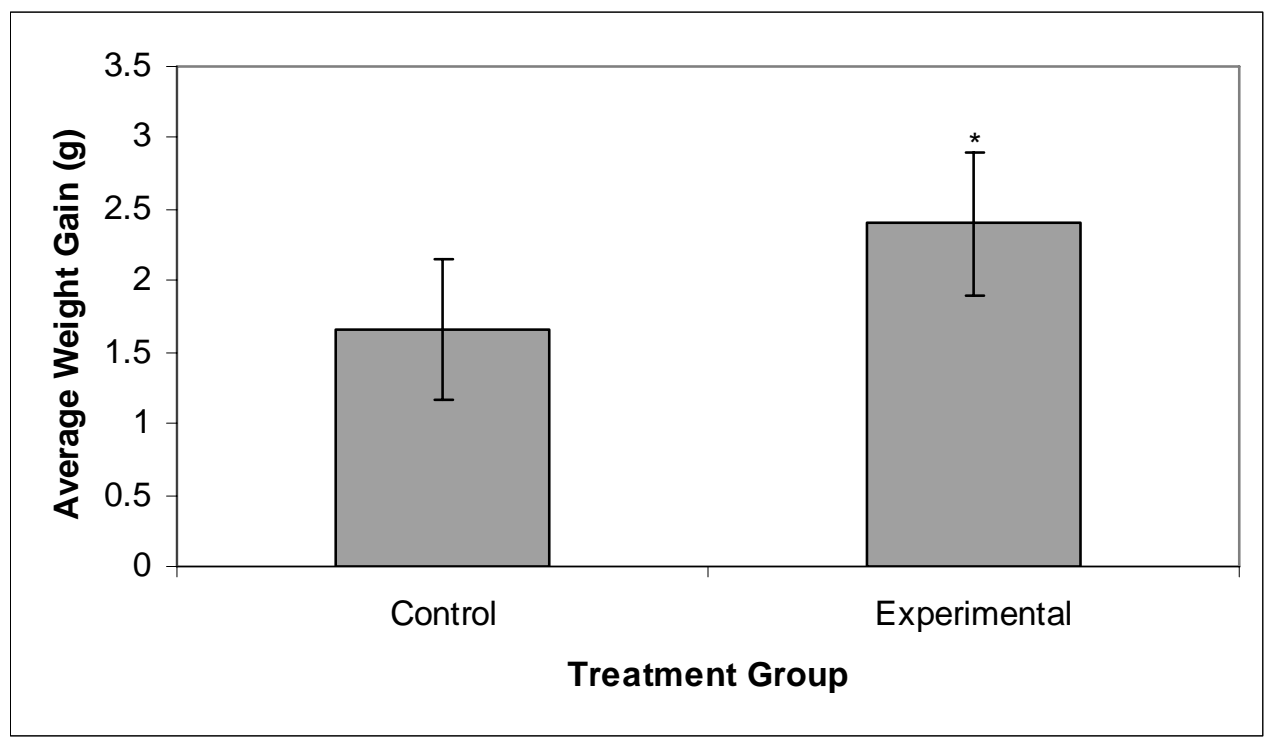

Figure 4. Average weight gain of control and experimental mice over a four-week period. Mice received fifty-five grams of food weekly and one hundred milliliters of water every two days. ${ }^{*} A$ significant difference existed between the treatment groups $(p<0.05)$.

the bedding under the sipper tubes to be wet on days that water consumption was excessive for the experimental mice.

The hypothesis of this experiment was supported, but the data obtained to make such conclusions could be improved by making several procedural modifications. One adjustment could be made with the type or placement of the water bottles. If the bottles could be placed in a different location less susceptible to the accumulation of bedding or the bottle possessed a different nozzle that would prevent excess water from being wasted when being brushed against, more accurate water consumption measure measurement could be made. Reducing the amount of bedding in each cage is also a solution if sanitary conditions within the cage could be maintained. Also, to be more certain that L-glutamine enhanced muscle growth, we could have weighed each individual gastrocnemius muscle. The average weight increase could be due to enhanced growth of muscle, but other factors may also contribute to increased overall body weight.

In future studies, measuring the mass of the gastrocnemius muscle alone could improve the content of the conclusion that may be made. The effect of increased muscle mass on the amount of weight gain experienced could be determined, and the relationship between contractile force and muscle size could be normalized more efficiently in calculations. By dividing the contractile force by the weight of the gastrocnemius muscle, the data used to determine a significant statistical difference between the contractile forces of the experimental and control mice would express better correlation within each group. If it could be effectively shown that larger muscles were formed in the experimental mice, than the conclusion that L-glutamine supplementation increased muscle mass leading to a greater muscle contractile force could be proved more successfully. Also, future research could implement the concept of exercising into the equation. Many human individuals, who may consume Lglutamine with the intentions of increasing their muscle mass and strength, exercise as well. Further research could test the effects of using an L-glutamine supplement along with exercising verses using only an Lglutamine supplement without exercising. By being able to test the use of L-glutamine supplementation in settings that may mimic its use in actual life situations, then the true effects of L-glutamine supplementation may be known. 


\section{ACKNOWLEDGEMENTS}

The authors thank the Department of Biology, Viterbo University, La Crosse, Wisconsin.

\section{REFERENCES}

1. Bickel, S.C., Slade, J.M., Warren, G.L., Dudley, G.A. (2003). "Fatigability and Variable-Frequency Train Stimulation of Human Skeletal Muscles," Physical Therapy, 83(4), 366-373.

2. Saladin, K.S. (2001). Anatomy and Physiology: The Unity of Form and Function. (2 ${ }^{\text {nd }}$ ed.). pp. 420-438.

3. Garlick, P.J. (2001). "Assessment of the Safety of Glutamine and Other Amino Acids," Glutamine Metabolism: Nutritional and Clinical Significance, 131, pp. 2556S-2561S.

4. Newsholme, P. (2001). "Why is LGlutamine Metabolism Important to Cells of the Immune System in Health, Post-injury, Surgery or Infection?" Glutamine Metabolism: Nutritional and Clinical Significance, 131, pp. 2515S2522S.17.

5. Lobley, G.E., Simone, H.O., McNeil, C.J. (2001). "Glutamine in Animal Science and Production," Glutamine Metabolism: Nutritional and Clinical Significance, 131, pp. 2525S-2531S.

6. Young, V.R., Ajami, A.M. (2001). "Glutamine: The Emperor and His Clothes," Glutamine Metabolism: Nutritional and Clinical Significance, 131, pp. 2449S-2459S.

7. Wilmore, D.W., Rombeau, J.L. (2001). "Introduction to the Symposium Proceedings," Glutamine Metabolism: Nutritional and Clinical Significance, 131, pp. 2447S-2448S.

8. Svanberg, E., Moller-Loswick, A., Matthews, P.E., Korner, U., Lundholm, K. (2001). "The effect of glutamine on protein balance and amino acid flex across arm and leg tissue in healthy volunteers," Clinical Physiology, 21(4), pp. 478-489.

9. Rennie, M.J., Bowtell, J.L., Bruce, M., Khogali S.E. (2001). "Interaction between Glutamine Availability and Metabolism of Glycogen, Tricarboxylic Acid Cycle Intermediates and Glutathione," Glutamine Metabolism:
Nutritional and Clinical Significance, 131, pp. 2488S-2490S.

10. Wakisaka, S., Ohshima, Y., Ogawa, M., Tochikura, T., Tachiki, T. (1993). "Characteristics and Efficiency of Glutamine Production by Coupling of a Bacterial Glutamine Synthetase Reaction with the Alcoholic Fermentation System of Baker's Yeast," Applied and Environmental Microbiology, 64(8), pp. 2952-2957.

11. Latham, N.K., Anderson, G.S., Reid, I.R. (2003). "Effects of Vitamin D Supplementation on Strength, Physical Performance, and Falls in Older Persons: A Systematic Review," American Geriatrics Society, 51, pp. 1219-1226.

12. Neu, J. (2001). "Glutamine in the Fetus and Critically III Low Birth Weight Neonate: Metabolism and Mechanism of Action," Glutamine Metabolism: Nutritional and Clinical Significance, 131, pp. 2585S-2589S.

13. Labow, B.I., Souba, W.W. (2000). "Glutamine," World Journal of Surgery, 24, pp. 1503-1513.

14. Wilmore, D.W. (2001). "The Effect of Glutamine Supplementation in Patients Following Elective Surgery and Accidental Injury," Glutamine Metabolism: Nutritional and Clinical Significance, 131, pp. 2543S-2549S.

15. Salleh, M., Ardaw, M., Majzoub, M.F. (1991). "Glutamine Metabolism in Skeletal Muscle of Septic Rats," Metabolism: Clinical and Experimental, 40, pp.155-164.

16. Nuutila, P., Kalliokoski, K., (2000). "Use of position emission tomography in the assessment of skeletal muscle and tendon metabolism and perfusion," Scandinavian Journal of Medicine and Science in Sports, 10, pp. 346-350.

17. Freireich, E.J. (1966). Cancer Chemotherapy Reports 50(4), pp. 219244.

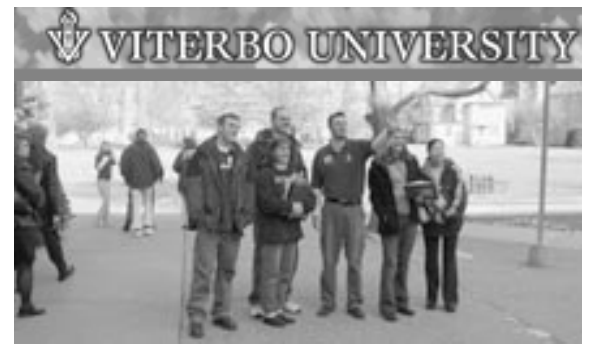




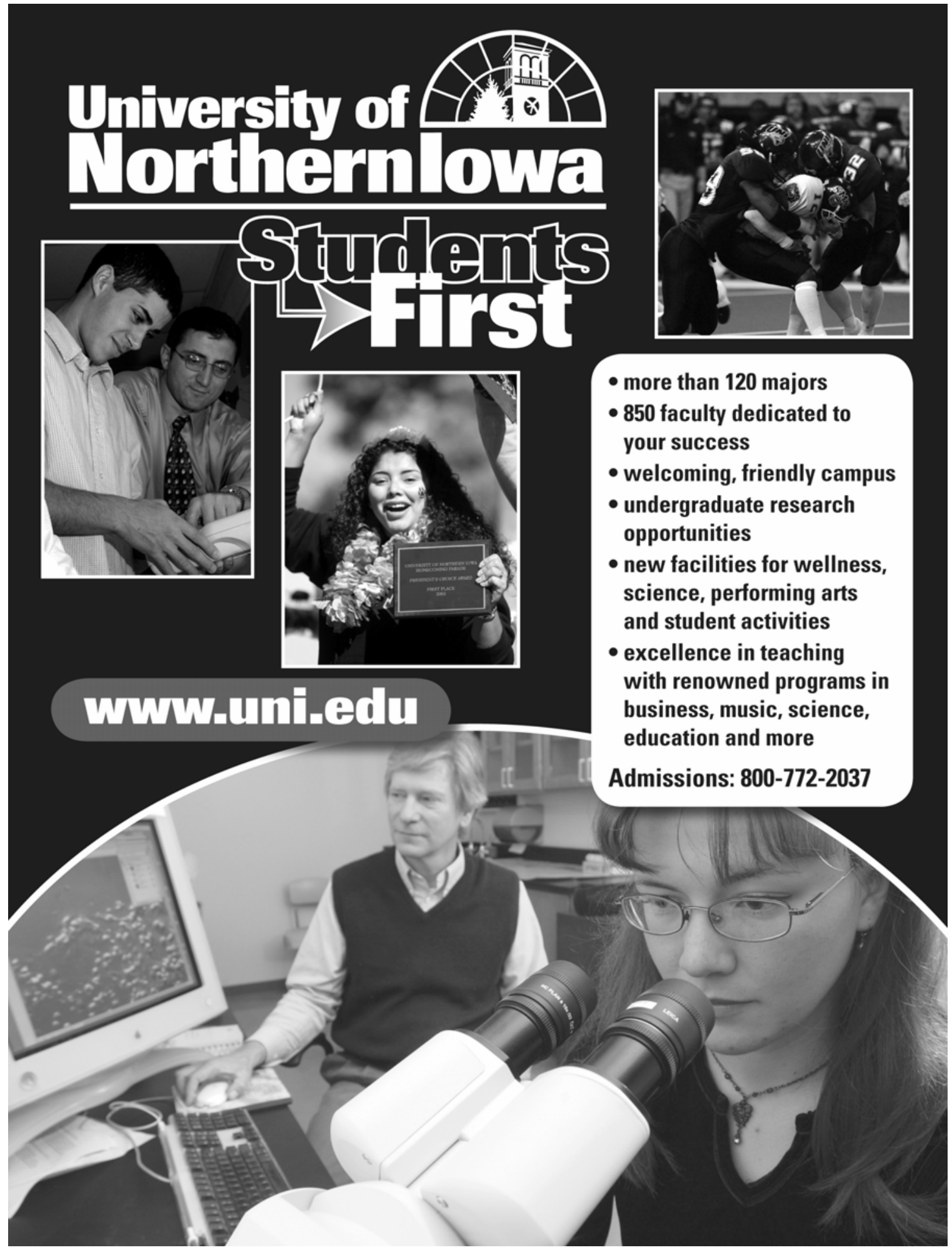

